PROCEEDINGS OF THE

AMERICAN MATHEMATICAL SOCIETY

Volume 138, Number 4, April 2010, Pages 1287-1293

S 0002-9939(09)10257-5

Article electronically published on December 3, 2009

\title{
THE SINGULAR EXTREMAL SOLUTIONS OF THE BI-LAPLACIAN WITH EXPONENTIAL NONLINEARITY
}

\author{
AMIR MORADIFAM
}

(Communicated by Matthew J. Gursky)

Abstract. Consider the problem

$$
\begin{cases}\Delta^{2} u=\lambda e^{u} & \text { in } B \\ u=\frac{\partial u}{\partial n}=0 & \text { on } \partial B\end{cases}
$$

where $B$ is the unit ball in $\mathbb{R}^{N}$ and $\lambda$ is a parameter. Unlike the Gelfand problem the natural candidate $u=-4 \ln (|x|)$, for the extremal solution, does not satisfy the boundary conditions, and hence showing the singular nature of the extremal solution in large dimensions close to the critical dimension is challenging. Recently a computer-assisted proof was used to show that the extremal solution is singular in dimensions $13 \leq N \leq 31$. Here by an improved Hardy-Rellich inequality we overcome this difficulty and give a simple mathematical proof to show that the extremal solution is singular in dimensions $N \geq 13$.

\section{INTRODUCTION}

Consider the fourth-order elliptic problem

$$
\begin{cases}\Delta^{2} u=\lambda e^{u} & \text { in } B \\ u=\frac{\partial u}{\partial n}=0 & \text { on } \partial B\end{cases}
$$

where $B$ is the unit ball in $\mathbb{R}^{N}, N \geq 1, n$ is the exterior unit normal vector and $\lambda \geq 0$ is a parameter. This problem is the fourth-order analogue of the classical Gelfand problem (see [2], 4], and 9]). Recently, many authors have become interested in fourth-order equations, and interesting results can be found in [1, 2, [3, [5], [8], 10], 11] and the references cited therein. In [1], Arioli et al. studied the problem (11) and showed that for each dimension $N \geq 1$ there exists a $\lambda^{*}>0$ such that for every $0<\lambda<\lambda^{*}$, there exists a smooth minimal (smallest) solution of (11), while for $\lambda>\lambda^{*}$ there is no solution even in a weak sense. Moreover, the branch $\lambda \mapsto u_{\lambda}(x)$ is increasing for each $x \in B$, and therefore the function $u^{*}(x):=\lim _{\lambda \nearrow^{*} \lambda^{*}} u_{\lambda}(x)$ can be considered as a generalized solution that corresponds to $\lambda^{*}$. Now the important question is whether $u^{*}$ is regular $\left(u^{*} \in L^{\infty}(B)\right)$ or singular $\left(u^{*} \notin L^{\infty}(B)\right)$. Even though there are similarities between (11) and the Gelfand problem, several tools which have been developed for the Gelfand problem

Received by the editors April 23, 2009.

2010 Mathematics Subject Classification. Primary 35J65; Secondary 35J40.

This work is supported by a Killam Predoctoral Fellowship and is part of the author's Ph.D. dissertation in preparation under the supervision of N. Ghoussoub.

(C)2009 American Mathematical Society 1287

Reverts to public domain 28 years from publication 
are no longer available for (11). In [5] the authors developed a new method to prove the regularity of the extremal solutions in low dimensions and showed that for $N \leq 12, u^{*}$ is regular. But unlike the Gelfand problem, the natural candidate $u=-4 \ln (|x|)$ for the extremal solution does not satisfy the boundary conditions, and hence showing the singular nature of the extremal solution in large dimensions close to the critical dimension is challenging. Dávila et al. [5 used a computerassisted proof to show that the extremal solution is singular in dimensions $13 \leq$ $N \leq 31$ while they gave a mathematical proof in dimensions $N \geq 32$. In this paper we introduce a unified mathematical approach to deal with this problem and show that for $N \geq 13$, the extremal solution is singular. One of our main tools is an improved Hardy-Rellich inequality that follows from the recent result of Ghoussoub-Moradifam about the improved Hardy and Hardy-Rellich inequalities developed in [7] and [6].

\section{AN IMPROVED HARDY-ReLLICH INEQUALITY}

In this section we shall prove an improvement of the classical Hardy-Rellich inequality, which will be used to prove our main result in Section 3. It relies on the results of Ghoussoub-Moradifam in [6] which provide necessary and sufficient conditions for such inequalities to hold. At the heart of this characterization is the following notion of a Bessel pair of functions.

Definition 1. Assume that $B$ is a ball of radius $R$ in $\mathbb{R}^{N}, V, W \in C^{1}(0,1)$, and $\int_{0}^{R} \frac{1}{r^{N-1} V(r)} d r=+\infty$. We say that the couple $(V, W)$ is a Bessel pair on $(0, R)$ if the ordinary differential equation

$$
\left(\mathrm{B}_{V, W}\right) \quad y^{\prime \prime}(r)+\left(\frac{N-1}{r}+\frac{V_{r}(r)}{V(r)}\right) y^{\prime}(r)+\frac{W(r)}{V(r)} y(r)=0
$$

has a positive solution on the interval $(0, R)$.

Theorem 2.1 (Ghoussoub-Moradifam 6]). Let $V$ and $W$ be positive radial $C^{1}$ functions on $B \backslash\{0\}$, where $B$ is a ball centered at zero with radius $R$ in $\mathbb{R}^{N}$ ( $N \geq$ 1) such that $\int_{0}^{R} \frac{1}{r^{N-1} V(r)} d r=+\infty$ and $\int_{0}^{R} r^{N-1} V(r) d r<+\infty$. The following statements are then equivalent:

(1) $(V, W)$ is a Bessel pair on $(0, R)$ and $\beta(V, W ; R) \geq 1$.

(2) $\int_{B} V(x)|\nabla u|^{2} d x \geq \int_{B} W(x) u^{2} d x$ for all $u \in C_{0}^{\infty}(B)$.

(3) If $\lim _{r \rightarrow 0} r^{\alpha} V(r)=0$ for some $\alpha<N-2$ and $W(r)-\frac{2 V(r)}{r^{2}}+\frac{2 V_{r}(r)}{r}-$ $V_{r r}(r) \geq 0$ on $(0, R)$, then the above are equivalent to

$$
\begin{array}{r}
\int_{B} V(x)|\Delta u|^{2} d x \geq \int_{B} W(x)|\nabla u|^{2} d x+(N-1) \int_{B}\left(\frac{V(x)}{|x|^{2}}-\frac{V_{r}(|x|)}{|x|}\right)|\nabla u|^{2} d x \\
\quad \text { for all } u \in C_{0}^{\infty}(B) .
\end{array}
$$

As an application we have the following improvement of the classical HardyRellich inequality. 
Theorem 2.2. Let $N \geq 5$ and $B$ be the unit ball in $\mathbb{R}^{N}$. Then the following improved Hardy-Rellich inequality holds for all $u \in C_{0}^{\infty}(B)$ :

$$
\begin{aligned}
\int_{B}|\Delta u|^{2} \geq & \frac{(N-2)^{2}(N-4)^{2}}{16} \int_{B} \frac{u^{2}}{\left(|x|^{2}-\frac{9}{10}|x|^{\frac{N}{2}+1}\right)\left(|x|^{2}-|x|^{\frac{N}{2}}\right)} \\
& +\frac{(N-1)(N-4)^{2}}{4} \int_{B} \frac{u^{2}}{|x|^{2}\left(|x|^{2}-|x|^{\frac{N}{2}}\right)} .
\end{aligned}
$$

As a consequence, the following improvement of the classical Hardy-Rellich inequality holds:

$$
\int_{B}|\Delta u|^{2} \geq \frac{N^{2}(N-4)^{2}}{16} \int_{B} \frac{u^{2}}{|x|^{2}\left(|x|^{2}-|x|^{\frac{N}{2}}\right)} .
$$

Proof. Let $\varphi:=r^{-\frac{N}{2}+1}-\frac{9}{10}$. Since

$$
-\frac{\varphi^{\prime \prime}+\frac{(N-1)}{r} \varphi^{\prime}}{\varphi}=\frac{(N-2)^{2}}{4} \cdot \frac{1}{r^{2}-\frac{9}{10} r^{\frac{N}{2}+1}},
$$

$\left(1, \frac{(N-2)^{2}}{4} \frac{1}{r^{2}-\frac{9}{10} r^{\frac{N}{2}+1}}\right)$ is a Bessel pair on $(0,1)$. By Theorem 2.1 the following inequality holds for all $u \in C_{0}^{\infty}(B)$ :

$$
\int_{B}|\Delta u|^{2} d x \geq \frac{(N-2)^{2}}{4} \int_{B} \frac{|\nabla u|^{2}}{|x|^{2}-\frac{9}{10}|x|^{\frac{N}{2}+1}}+(N-1) \int_{B} \frac{|\nabla u|^{2}}{|x|^{2}} .
$$

Let $V(r):=\frac{1}{r^{2}-\frac{9}{10} r^{\frac{N}{2}+1}}$. Then

$$
\frac{V_{r}}{V}=-\frac{2}{r}+\frac{9}{10}\left(\frac{N-2}{2}\right) \frac{r^{\frac{N}{2}-2}}{1-\frac{9}{10} r^{\frac{N}{2}-1}} \geq-\frac{2}{r}
$$

and $\psi(r)=r^{-\frac{N}{2}+2}-1$ is a positive super-solution for the ODE

$$
y^{\prime \prime}+\left(\frac{N-1}{r}+\frac{V_{r}}{V}\right) y^{\prime}(r)+\frac{W_{1}(r)}{V(r)} y=0,
$$

where

$$
W_{1}(r)=\frac{(N-4)^{2}}{4\left(r^{2}-r^{\frac{N}{2}}\right)\left(r^{2}-\frac{9}{10} r^{\frac{N}{2}+1}\right)} .
$$

Hence the ODE (6) actually has a positive solution, and by Theorem 2.1] we have

$$
\int_{B} \frac{|\nabla u|^{2}}{|x|^{2}-\frac{9}{10}|x|^{\frac{N}{2}+1}} \geq\left(\frac{N-4}{2}\right)^{2} \int_{B} \frac{u^{2}}{\left(|x|^{2}-\frac{9}{10}|x|^{\frac{N}{2}+1}\right)\left(|x|^{2}-|x|^{\frac{N}{2}}\right)} .
$$

Similarly

$$
\int_{B} \frac{|\nabla u|^{2}}{|x|^{2}} \geq\left(\frac{N-4}{2}\right)^{2} \int_{B} \frac{u^{2}}{|x|^{2}\left(|x|^{2}-|x|^{\frac{N}{2}}\right)} .
$$

Combining the above two inequalities with (4) we get (2). 


\section{MAIN RESUlts}

In this section we shall prove that the extremal solution $u^{*}$ of the problem (11) is singular in dimensions $N \geq 13$. The next lemma will be our main tool to guarantee that $u^{*}$ is singular for $N \geq 13$. The proof is based on an upper estimate by a singular stable sub-solution.

Lemma 3.1. Suppose there exist $\lambda^{\prime}>0$ and a radial function $u \in H^{2}(B) \cap$ $W_{l o c}^{4, \infty}(B \backslash\{0\})$ such that

$$
\begin{gathered}
\Delta^{2} u \leq \lambda^{\prime} e^{u} \quad \text { for all } 0<r<1, \\
u(1)=0, \quad \frac{\partial u}{\partial n}(1)=0, \\
u \notin L^{\infty}(B),
\end{gathered}
$$

and

$$
\beta \int_{B} e^{u} \varphi^{2} \leq \int_{B}(\Delta \varphi)^{2} \text { for all } \varphi \in C_{0}^{\infty}(B),
$$

for some $\beta>\lambda^{\prime}$. Then $u^{*}$ is singular and

$$
\lambda^{*} \leq \lambda^{\prime}
$$

Proof. By Lemma 2.6 in [5], we have (13). Define

$$
\alpha:=\ln \left(\frac{\lambda^{\prime}}{\gamma \lambda^{*}}\right),
$$

where $\frac{\lambda^{\prime}}{\beta}<\gamma<1$ and let $\bar{u}:=u+\alpha$. We claim that

$$
u^{*} \leq \bar{u} \text { in } B .
$$

To prove this, we shall show that for $\lambda<\lambda^{*}$,

$$
u_{\lambda} \leq \bar{u} \text { in } B .
$$

Indeed, we have

$$
\Delta^{2}(\bar{u})=\Delta^{2}(u) \leq \lambda^{\prime} e^{u}=\lambda^{\prime} e^{-\alpha} e^{\bar{u}}=\gamma \lambda^{*} e^{\bar{u}} .
$$

To prove (15) it suffices to prove it for $\gamma \lambda^{*}<\lambda<\lambda^{*}$. Fix such a $\lambda$ and assume that (15) is not true. Let

$$
R_{1}:=\sup \left\{0 \leq R \leq 1 \mid u_{\lambda}(R)=\bar{u}(R)\right\} .
$$

Since $\bar{u}(1)=\alpha>0=u_{\lambda}(1)$, we have $0<R_{1}<1, u_{\lambda}\left(R_{1}\right)=\bar{u}\left(R_{1}\right)$, and $u_{\lambda}^{\prime}\left(R_{1}\right) \leq$ $\bar{u}^{\prime}\left(R_{1}\right)$. Now consider the following problem:

$$
\begin{cases}\Delta^{2} u=\lambda e^{u} & \text { in } \Omega, \\ u=u_{\lambda}\left(R_{1}\right) & \text { on } \partial \Omega, \\ \frac{\partial u}{\partial n}=u_{\lambda}^{\prime}\left(R_{1}\right) & \text { on } \partial \Omega .\end{cases}
$$

Obviously $u_{\lambda}$ is a solution to the above problem while $\bar{u}$ is a sub-solution to the same problem. Moreover, $\bar{u}$ is stable since

$$
\lambda<\lambda^{*}
$$

and hence

$$
\lambda e^{\bar{u}} \leq \lambda^{*} e^{\alpha} e^{u}=\frac{\lambda^{\prime}}{\gamma} e^{u}<\beta e^{u}
$$


We deduce $\bar{u} \leq u_{\lambda}$ in $B_{R_{1}}$, which is impossible, since $\bar{u}$ is singular while $u_{\lambda}$ is smooth. This establishes (15). From (15) and the above two inequalities we have

$$
\lambda^{*} e^{u^{*}} \leq \lambda^{*} e^{a} e^{u}=\frac{\lambda^{\prime}}{\gamma} e^{u} .
$$

Since $\frac{\lambda^{\prime}}{\gamma}<\beta$

$$
\inf _{\varphi \in C_{0}^{\infty}(B)} \frac{\int_{B}(\Delta \varphi)^{2}-\lambda^{*} e^{u^{*}}}{\int_{B} \varphi^{2}}>0 .
$$

This is not possible if $u^{*}$ is a smooth solution.

In the following, for each dimension $N \geq 13$ we shall construct $u$ satisfying all the assumptions of Lemma 3.1. Define

$$
w_{m}:=-4 \ln (r)-\frac{4}{m}+\frac{4}{m} r^{m}, \quad m>0,
$$

and let $H_{N}:=\frac{N^{2}(N-4)^{2}}{16}$. Now we are ready to prove our main result.

Theorem 3.2. The following upper bounds on $\lambda^{*}$ hold in large dimensions.

(1) If $N \geq 32$, then Lemma 3.1 holds with $u:=w_{2}, \lambda_{N}^{\prime}=8(N-2)(N-4) e^{2}$ and $\beta=H_{N}>\lambda_{N}^{\prime}$.

(2) If $13 \leq N \leq 31$, then Lemma 3.1 holds with $u:=w_{3.5}$ and $\lambda_{N}^{\prime}<\beta_{N}$ given in Table 1 in 7 .

The extremal solution is therefore singular for dimensions $N \geq 13$.

Proof. 1) Assume first that $N \geq 32$. Then

and

$$
8(N-2)(N-4) e^{2}<\frac{N^{2}(N-4)^{2}}{16}
$$

Moreover,

$$
\Delta^{2} w_{2}=8(N-2)(N-4) \frac{1}{r^{4}} \leq 8(N-2)(N-4) e^{2} e^{w_{2}} .
$$

$$
8(N-2)(N-4) e^{2} \int_{B} e^{w_{2}} \varphi^{2} \leq H_{n} \int_{B} e^{-4 \ln (|x|)} \varphi^{2}=H_{n} \int_{B} \frac{\varphi^{2}}{|x|^{2}} \leq \int_{B}|\Delta \varphi|^{2} .
$$

Thus it follows from Lemma 3.1 that $u^{*}$ is singular and $\lambda^{*} \leq 8(N-2)(N-4) e^{2}$.

2 ) Assume $13 \leq N \leq 31$. We shall show that $u=w_{3.5}$ satisfies the assumptions of Lemma 3.1 for each dimension $13 \leq N \leq 31$. Using Maple, for each dimension $13 \leq N \leq 31$ one can verify that inequality (9) holds for the $\lambda_{N}^{\prime}$ given by Table 1 . Then, by using Maple again, we show that there exists $\beta_{N}>\lambda_{N}^{\prime}$ such that

$$
\begin{aligned}
\frac{(N-2)^{2}(N-4)^{2}}{16} & \frac{1}{\left(|x|^{2}-0.9|x|^{\frac{N}{2}+1}\right)\left(|x|^{2}-|x|^{\frac{N}{2}}\right)} \\
+\frac{(N-1)(N-4)^{2}}{4} & \frac{1}{|x|^{2}\left(|x|^{2}-|x|^{\frac{N}{2}}\right)} \geq \beta_{N} e^{w_{3.5} .}
\end{aligned}
$$

The above inequality and improved Hardy-Rellich inequality (2) guarantee that the stability condition (12) holds for $\beta_{N}>\lambda^{\prime}$. Hence by Lemma 3.1 the extremal solution is singular for $13 \leq N \leq 31$. The values of $\lambda_{N}$ and $\beta_{N}$ are shown in Table 1 .

Remark 3.3. The values of $\lambda_{N}^{\prime}$ and $\beta_{N}$ in Table 1 are not optimal. 
TABLE 1. Summary

\begin{tabular}{ccc}
\hline \hline $\mathrm{N}$ & $\lambda_{N}^{\prime}$ & $\beta_{N}$ \\
\hline$N \geq 32$ & $8(N-2)(N-4) e^{2}$ & $H_{n}$ \\
31 & 20000 & 86900 \\
30 & 18500 & 76500 \\
29 & 17000 & 67100 \\
28 & 16000 & 58500 \\
27 & 14500 & 50800 \\
26 & 13500 & 43870 \\
25 & 12200 & 37630 \\
24 & 11100 & 32050 \\
23 & 10100 & 27100 \\
22 & 9050 & 22730 \\
21 & 8150 & 18890 \\
20 & 7250 & 15540 \\
19 & 6400 & 12645 \\
18 & 5650 & 10155 \\
17 & 4900 & 8035 \\
16 & 4230 & 6250 \\
15 & 3610 & 4765 \\
14 & 3050 & 3545 \\
13 & 2525 & 2560 \\
\hline
\end{tabular}

Remark 3.4. The improved Hardy-Rellich inequality (2) is crucial to prove that $u^{*}$ is singular in dimensions $N \geq 13$. Indeed by the classical Hardy-Rellich inequality and $u:=w_{3.5}$, Lemma 3.1 only implies that $u^{*}$ is singular in dimensions $N \geq 22$.

\section{ACKNOWLEDGMENTS}

I would like to thank Professor Nassif Ghoussoub, my supervisor, for his valuable suggestions, constant support, and encouragement. I would also like to thank the anonymous referee for a careful reading of this paper.

\section{REFERENCES}

[1] G. Arioli, F. Gazzola, H.-C. Grunau, E. Mitidieri, A semilinear fourth order elliptic problem with exponential nonlinearity. SIAM J. Math. Anal. 36 (2005), no. 4, 1226-1258. MR2139208 (2006c:35070)

[2] H. Brezis, J.L. Vazquez, Blow-up solutions of some nonlinear elliptic problems. Rev. Mat, Univ. Compl. Madrid 10 (1997), 443-468. MR1605678 (99a:35081)

[3] C. Cowan, P. Esposito, N. Ghoussoub, A. Moradifam, The critical dimension for a fourth order elliptic problem with singular nonlinearity, Arch. Ration. Mech. Anal., to appear.

[4] M. G. Crandall and P. H. Rabinowitz, Some continuation and variational methods for positive solutions of nonlinear elliptic eigenvalue problems, Arch. Ration. Mech. Anal. 58 (1975), 207218. MR0382848 (52:3730)

[5] J. Dávila, L. Dupaigne, I. Guerra, M. Montenegro, Stable solutions for the bilaplacian with exponential nonlinearity, SIAM J. Math. Anal. 39 (2007), 565-592. MR 2338421(2008h:35053)

[6] N. Ghoussoub, A. Moradifam, Bessel pairs and optimal Hardy and Hardy-Rellich inequalities, submitted.

[7] N. Ghoussoub, A. Moradifam, On the best possible remaining term in the Hardy inequality, Proc. Natl. Acad. Sci. USA 105 (2008), no. 37, 13746-13751. MR2443723 (2009i:26031) 
[8] Z. Gui, J. Wei, On a fourth order nonlinear elliptic equation with negative exponent, SIAM J. Math. Anal. 40 (2009), 2034-2054. MR2471911

[9] F. Mignot, J.P. Puel, Solution radiale singulière de $-\Delta u=\lambda e^{u}$. C. R. Acad. Sci. Paris Ser. I Math. 307 (1988), no. 8, 379-382. MR965802 (89h:35119)

[10] A. Moradifam, On the critical dimension of a fourth order elliptic problem with negative exponent, Journal of Differential Equations 248 (2010), 594-616.

[11] J. Wei, Asymptotic behavior of a nonlinear fourth order eigenvalue problem, Comm. Partial Differential Equations 21 (1996), 1451-1467. MR.1410837(97h:35066)

Department of Mathematics, University of British Columbia, Vancouver, BC, Canada V6T 1 Z2

E-mail address: a.moradi@math.ubc.ca 